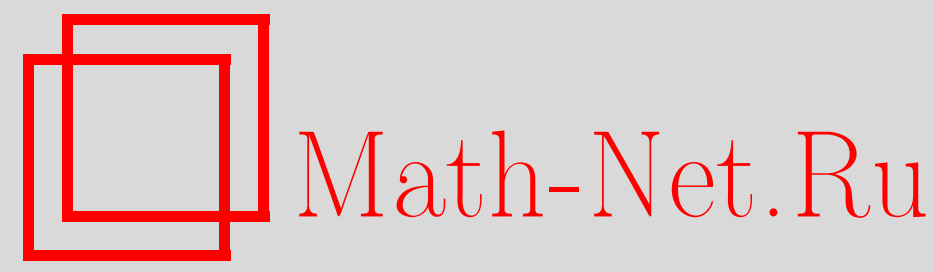

Ю. В. Егоров, В. А. Кондратьев, О. А. Олейник, Асимптотическое поведение решений нелинейных эллиптических и параболических систем в цилиндрических областях, Матем. сб., 1998, том 189, номер 3, 45-68

DOI: https://doi.org/10.4213/sm304

Использование Общероссийского математического портала Math-Net.Ru подразумевает, что вы прочитали и согласны с пользовательским соглашением

http://www . mathnet.ru/rus/agreement

Параметры загрузки:

IP : 54.172 .240 .79

26 апреля 2023 г., 18:17:20 
УДК 517.9

\author{
Ю.В. Егоров, В.А. Кондратьев, О.А. Олейник
}

\title{
Асимптотическое поведение решений нелинейных эллиптических и параболических систем в цилиндрических областях
}

\begin{abstract}
Статья посвящена изучению асимптотики решений слабонелинейных эллиптических и параболических систем уравнений второго порядка. В частности, изучается поведение при $t \rightarrow+\infty$ решения нелинейного параболического уравнения второго порядка, удовлетворяющего краевьм условиям Неймана на границе ограниченной липшицевой области. Доказательства основаны на теореме об асимптотической эквивалентности двух систем обыкновенных дифференциальных уравнений.

Библиографория: 8 названий.
\end{abstract}

Статья посвящена изучению асимптотических свойств решений некоторого класса параболических и эллиптических уравнений второго порядка и систем уравнений такого же типа. Этот класс уравнений называется полулинейные уравнения. Основное внимание уделено сушествованию и асимптотике положительных решений, хотя изучаются и любые, возможно, знакопеременные решения. Полученные результаты обобщают и уточняют результаты работ [1], [2]. Приведены примеры, показывающие, что методы настоящей работы позволяют рассмотреть уравнения и системы порядка выше второго.

Основой метода является некоторый результат из теории систем обыкновенных дифференциальных уравнений.

Рассмотрим две системы обыкновенных дифференциальных уравнений:

$$
\dot{x}=A x+B(t) x+f(x, t)+\varphi_{1}(t)
$$

и

$$
\dot{x}=A x+B(t) x+f(x, t)+\varphi_{2}(t),
$$

где $\dot{x} \equiv \frac{d x}{d t}, x \in \mathbb{R}^{n}, A=\left(a_{i j}\right)_{n \times n}-$ постоянная матрица, $B(t)=\left(b_{i j}(t)\right)_{n \times n}-$ матрица, элементы которой непрерывны при $t \geqslant 0$ и

$$
\lim _{t \rightarrow+\infty} B(t)=0 \text {. }
$$

Векторзначная функция $f(x, t)$ определена при $t \geqslant 0,\|x\|=\max _{i \leqslant n}\left|x_{i}\right| \leqslant a$, $a=$ const $>0$, где она дифференцируема по $x$ и измерима по $t$,

$$
\lim _{x \rightarrow 0} \frac{\partial f_{i}}{\partial x_{j}}=0, \quad i, j=1, \ldots, n,
$$

Работа вьполнена при частичной поддержке Российского фонда фундаментальных исследований (грант № 96-15-96177).

(C) Ю. В. ЕГоров, В. А. КондРАтьев, О. А. ОЛЕйник 
равномерно по $t \in[0, \infty)$.

Предполагается, что

$$
\int_{0}^{\infty}\left\|\varphi_{1}-\varphi_{2}\right\|^{2} e^{2 b t} d t<\infty
$$

где $b>0$ - некоторая постоянная.

Пусть матрица $A$ в правой части систем $(1),(2)$ не имеет точек спектра на прямой $\operatorname{Re} \lambda=-b$.

Если все эти предположения выполнены, то имеет место следуюшая теорема.

Tеорема 1. Существует $\rho_{0}=$ const $>0$ такое, что для любого решения $x(t)$ системь (1), удовлетворяющего условию:

$$
\|x(t)\| \leqslant \rho_{0}, \quad 0 \leqslant t<\infty,
$$

найдется решение системы (2) $\widehat{x}(t)$ такое, что

$$
\|x(t)-\widehat{x}(t)\|=o\left(e^{-b t}\right), \quad \int_{0}^{\infty}\|x(t)-\widehat{x}(t)\|^{2} e^{2 b t} d t<\infty .
$$

ДокаЗАТЕЛЬСТво. Пусть $x(t)$ - ограниченное на $[0, \infty)$ решение системы $(1)$ и пусть

$$
\rho_{1}=\sup _{t \geqslant 0}\|x(t)\|
$$

Предположим, что $\rho_{1}<a / 2$, где постоянная $a$ была определена выше. Сделаем в (2) замену переменных:

$$
x=x(t)+y .
$$

В результате получим систему уравнений для $y$ :

$$
\dot{y}=A y+B(t) y+f(x(t)+y, t)-f(x(t), t)+\varphi_{2}(t)-\varphi_{1}(t)
$$

или

$$
\dot{y}=A y+B(t) y+g(y, t)+\varphi(t),
$$

где $\varphi(t)=\varphi_{2}(t)-\varphi_{1}(t), g(y, t)=f(x(t)+y, t)-f(x(t), t)$. Заметим, что $g(y, t)$ дифференцируема по $y$, измерима по $t$ при $t \geqslant 0,\|y\| \leqslant a / 2$, а $g(0, t) \equiv 0$. Кроме того, в силу (4) и формулы Лагранжа

$$
\|g(y, t)\| \leqslant C\left(\rho_{1},\|y\|\right)\|y\|,
$$

причем $C\left(\rho_{1},\|y\|\right) \rightarrow 0$, если $\rho_{1} \rightarrow 0,\|y\| \rightarrow 0$. Постоянная $C$ в правой части неравенства (10) от $t$ не зависит.

Вектор-функция $\varphi(t)$ в $(9)$ такова, что

$$
\int_{0}^{\infty}\|\varphi(t)\|^{2} e^{2 b t} d t<\infty
$$

Рассмотрим множество $M\left(t_{1}\right)$ непрерывных при $t \geqslant t_{1}$ функций $z(t)$ таких, что

$$
\|z(t)\| \leqslant \frac{a}{2} e^{-b t} \text { при } t \geqslant t_{1}
$$


и

$$
\int_{t_{1}}^{\infty}\|z(t)\|^{2} e^{2 b t} d t \leqslant \frac{a}{2}
$$

Это множество является полным метрическим пространством с метрикой:

$$
\rho\left(z_{1}, z_{2}\right)=\sup _{t \geqslant t_{1}}\left(\left\|z_{1}-z_{2}\right\| e^{b t}\right)+\left(\int_{t_{1}}^{\infty}\left\|z_{1}(t)-z_{2}(t)\right\|^{2} e^{2 b t} d t\right)^{1 / 2} .
$$

Рассмотрим на $M$ оператор $T$ такой, что

$$
T z=R B(t) z+R g(z, t)+R \varphi(t)
$$

где оператор $R$ определяется следующим образом. Пусть $\psi(t),-\infty<t<+\infty$, такова, что

$$
\int_{-\infty}^{+\infty}\|\psi(t)\|^{2} e^{2 b t} d t<\infty
$$

Положим $R_{t_{1}} \psi \equiv \psi(t)$, если $t \geqslant t_{1}, R_{t_{1}}(\psi)=0$, если $t<t_{1}$. Рассмотрим систему обыкновенных дифференциальных уравнений

$$
\frac{d u}{d t}=A u+F(t)
$$

где

$$
\int_{-\infty}^{+\infty}\|F\|^{2} e^{2 b t} d t<\infty
$$

Эта система имеет единственное решение $u(t)$ такое, что

$$
\int_{-\infty}^{+\infty}\|u(t)\|^{2} e^{2 b t} d t<\infty
$$

и это решение удовлетворяет неравенству

$$
\int_{-\infty}^{+\infty}\|u(t)\|^{2} e^{2 b t} d t+\int_{-\infty}^{+\infty}\left\|\frac{d u}{d t}\right\|^{2} e^{2 b t} d t \leqslant c \int_{-\infty}^{+\infty}\|F(t)\|^{2} e^{2 b t} d t .
$$

Будем обозначать $u=S F, R=S R_{t_{1}}$.

Очевидно, что если $z \in M$, то оператор $T$ определен. Если $\rho_{1}$ достаточно мало и $t_{1}$ достаточно большое, то при всяком $z \in M$ имеем

$$
T z \in M \text {. }
$$

При этом $t_{1}$ зависит от $\varphi$ и от $B(t)$.

Покажем, что оператор $T$ сжимающий, если $\rho_{1}$ достаточно мало, a $t_{1}$ достаточно велико. В самом деле,

$$
T z_{1}-T z_{2}=R B(t)\left(z_{1}-z_{2}\right)+R\left[g\left(z_{1}, t\right)-g\left(z_{2}, t\right)\right]
$$

Так как

$$
\left\|g\left(z_{1}, t\right)-g\left(z_{2}, t\right)\right\| \leqslant\left\|z_{1}-z_{2}\right\| C\left(\left\|z_{1}\right\|,\left\|z_{2}\right\|\right),
$$


где $C\left(\xi_{1}, \xi_{2}\right) \rightarrow 0$ при $\xi_{1} \rightarrow 0, \xi_{2} \rightarrow 0$, то, выбрав $t_{1}$ достаточно большим, получим:

$$
\left\|g\left(z_{1}, t\right)-g\left(z_{2}, t\right)\right\| \leqslant \varepsilon\left|z_{1}-z_{2}\right|
$$

при любом $\varepsilon>0$ и $t \geqslant t_{1}(\varepsilon)$. Отсюда следует

$$
\int_{t_{1}}^{\infty} e^{2 b t}\left\|g\left(z_{1}, t\right)-g\left(z_{2}, t\right)\right\|^{2} d t \leqslant \varepsilon^{2} \int_{t_{1}}^{\infty} e^{2 b t}\left|z_{1}-z_{2}\right|^{2} d t \leqslant \varepsilon^{2} \rho^{2}\left(z_{1}, z_{2}\right) .
$$

Из (12) и (13), учитывая малость $\|B(t)\|$ при больших $t_{1}$, получим, что отображение $T$ является сжимающим. Следовательно, существует $z$-неподвижная точка оператора $T$, т.е.

$$
z=R B z+R g(z, t)+R \varphi(t)
$$

Отсюда

$$
\dot{z}=A z+B(t) z+g(z, t)+\varphi(t)
$$

т.е. $z(t)$ является решением уравнения (8) и

$$
z(t)=o\left(e^{-b t}\right)
$$

Таким образом, сушествует $\widehat{x}(t)$ - решение уравнения $(2)$ вида $\widehat{x}=x+o\left(e^{-b t}\right)$. Теорема доказана.

ЗАмечаниЕ. Решению уравнения (1), удовлетворяюшему условию (6), может соответствовать несколько решений уравнения (2), удовлетворяюших условию (7). Нетрудно показать, что если спектр матрицы $A$ лежит правее прямой $\operatorname{Re} \lambda=-b$, то каждому решению $X(t)$ системы (1), удовлетворяющему условию (6), соответствует единственное решение системы (2) такое, что справедливо равенство (8).

Теорема 1 будет сушественно использоваться при исследовании асимптотического поведения решений эллиптических и параболических уравнений в цилиндрических областях.

Рассмотрим систему уравнений:

$$
\frac{\partial u_{i}}{\partial t}-L_{i}\left(u_{i}\right)+f_{i}(u, t)=0, \quad u=\left(u_{1}, \ldots, u_{N}\right), \quad i=1, \ldots, N
$$

где

$$
L_{k} u \equiv \sum_{i, j=1}^{n} \frac{\partial}{\partial x_{i}} a_{i j}^{(k)}(x) \frac{\partial u}{\partial x_{j}}+\sum_{i=1}^{n} a_{i}^{(k)}(x) \frac{\partial u}{\partial x_{i}}
$$

Предполагается, что все операторы $L_{i}, i=1, \ldots, N$, равномерно эллиптичны в некоторой ограниченной области $\Omega$ с липшицевой границей, т.е.

$$
m_{1}|\xi|^{2} \leqslant \sum_{i, j=1}^{n} a_{i j}^{(k)}(x) \xi_{i} \xi_{j} \leqslant m_{2}|\xi|^{2}
$$

$m_{1}=$ const $>0,|\xi|^{2}=\sum_{i=1}^{n} \xi_{i}^{2}, x=\left(x_{1}, \ldots, x_{n}\right), x \in \Omega$. Кроме того, $a_{i j}^{(k)} \equiv a_{j i}^{(k)}$, все функции $a_{i j}^{(k)}, a_{i}^{(k)}$ измеримы и ограничены в $\Omega$. 
Пусть векторзначная функция $f(u, t)=\left(f_{1}, \ldots, f_{N}\right)$ определена при $\|u\| \leqslant a$, где $\|u\|=\max _{i \leqslant N}\left|u_{i}\right|, 0 \leqslant t<+\infty, f(0, t) \equiv 0$. Предполагается, что $f(u, t)$ непрерывна по $u$ и измерима по $t$ при фиксированном $u$. Кроме того, предполагается, что сушествуют производные $\frac{\partial f_{i}}{\partial u_{j}}, i, j=1, \ldots, N$, при $\|u\| \leqslant a, 0 \leqslant t<\infty$ и

$$
\lim _{u \rightarrow 0} \frac{\partial f_{i}}{\partial u_{j}}=0
$$

равномерно по $t$.

Будем рассматривать решение системы (14) в области $\Pi_{0}=\Omega \times[0, \infty)$, которое удовлетворяет краевому условию:

$$
\frac{\partial u_{i}}{\partial \nu_{i}}=0, \quad x \in \partial \Omega, \quad t>0, \quad i=1, \ldots, N
$$

где $\frac{\partial}{\partial \nu_{i}}-$ дифференцирование по направлению внешней конормали оператора $L_{i}$ :

$$
\frac{\partial u_{i}}{\partial \nu_{i}}=\sum_{k, j=1}^{n} a_{k j}^{(i)}(x) \cos \left(\vec{n}, x_{j}\right) \frac{\partial}{\partial x_{k}} .
$$

Здесь $\vec{n}$ - направление внешней нормали к $\partial \Omega$.

В качестве решения уравнения (15), удовлетворяющего условию (16), понимается обобшенное решение в стандартном определении.

Напомним определение обобщенного решения.

Будем обозначать: $\Pi_{a, b}=\Omega \times(a, b), \Pi_{a, \infty}=\Pi_{a}, \Gamma_{a, b}=\partial \Omega \times(a, b), \Gamma_{a}=\Gamma_{a, \infty}$. Вектор-функция $u(x, t)$ есть обобщенное решение уравнения (14), удовлетворяющее условию (16), если $u(x, t) \in W_{2}^{1}\left(\Pi_{a, b}\right) \cap L_{\infty}\left(\Pi_{a, b}\right)$ при любых $a, b, 0<a<$ $b<\infty$, и какова бы ни была вектор-функция $\psi(x, t) \in W_{2}^{1}\left(\Pi_{a, b}\right)$ имеет место тождество:

$$
\begin{aligned}
& \sum_{k=1}^{N} \int_{\Pi_{a, b}} \psi_{k} \frac{\partial u_{k}}{\partial t} d x d t+\sum_{k=1}^{N} \sum_{i, j=1}^{n} \int_{\Pi_{a, b}} a_{i j}^{(k)} \frac{\partial \psi_{k}}{\partial x_{i}} \frac{\partial u_{k}}{\partial x_{j}} d x d t \\
& -\sum_{k=1}^{N} \sum_{i=1}^{n} \int_{\Pi_{a, b}} a_{i}^{(k)}(x) \frac{\partial u_{k}}{\partial x_{i}} \psi_{k} d x d t+\int_{\Pi_{a, b}} \sum_{k=1}^{N} f_{k}(u, t) \psi_{k}(x, t) d x d t=0 .
\end{aligned}
$$

Из классических результатов о гладкости решений линейных параболических уравнений [3] следует, что $u(x, t)$ непрерьвна в $\bar{\Pi}_{0}$ при каждом $a>0$ и, более того, удовлетворяет условию Гёльдера в каждой замкнутой области $\bar{\Pi}_{a, b}$, где $0<a<$ $b<\infty$.

В дальнейшем будет использоваться следуюшее свойство решений линейных эллиптических задач. Рассмотрим краевую задачу:

$$
\begin{aligned}
& L_{k}^{*} u=\sum_{i, j=1}^{n} \frac{\partial}{\partial x_{i}} a_{i j}^{(k)} \frac{\partial u}{\partial x_{j}}-\sum_{i=1}^{n} \frac{\partial}{\partial x_{i}}\left(a_{i}^{(k)} u\right)=0, \quad x \in \Omega, \\
& \sum_{i, j=1}^{n} a_{i j}^{(k)} \frac{\partial u}{\partial x_{j}} \cos \left(\vec{n}, x_{i}\right)-\sum_{i=1}^{n} a_{i}^{(k)} u \cos \left(\vec{n}, x_{i}\right)=0, \quad x \in \partial \Omega
\end{aligned}
$$


где $\vec{n}$ - направление внешней нормали к $\partial \Omega$. Задача (18) является сопряженной задачей к задаче Неймана для оператора $L_{k}$ в $\Omega$ и, следовательно, имеет нетривиальное решение $u_{k 0}(x)$. Функции $u_{k 0}(x)$ являются непрерывными в $\bar{\Omega}$ и не обращаются в нуль в $\bar{\Omega}$. Будем считать, что $u_{k 0}(x)>0$ и

$$
\int_{\Omega} u_{k 0}(x) d x=1
$$

ТЕОРЕма 2. Если и $(x, t)$ - решение системы (14) - удовлетворяет краевому условию (16) и

$$
\lim _{t \rightarrow+\infty} u(x, t)=0
$$

mo

$$
u(x, t)=\chi(t)+v(x, t),
$$

әде $\chi(t)$ - некоторое решение системы:

$$
\dot{x}+f(x, t)=0
$$

а $v(x, t)$ таково, что

$$
\int_{\Pi_{\tau}} v^{2} d x d t=O\left(e^{-\alpha \tau}\right), \quad \alpha=\text { const }>0 .
$$

ДокАЗАТЕЛЬство. Зафиксируем $k(1 \leqslant k \leqslant N)$ и положим в $(17): \psi_{i}=0$, если $i \neq k, \psi_{k}(x, t)=\varepsilon^{-1} u_{k 0}(x), b=a+\varepsilon$. В результате получим:

$$
\begin{aligned}
& \varepsilon^{-1} \int_{\Pi_{a, a+\varepsilon}} u_{k 0}(x) \frac{\partial u_{k}}{\partial t} d x d t+\int_{\Pi_{a, a+\varepsilon}} \varepsilon^{-1} \sum_{i, j=1}^{n} a_{i j}^{(k)} \frac{\partial u_{k 0}}{\partial x_{i}} \frac{\partial u_{k}}{\partial x_{i}} d x d t \\
& \quad-\sum_{i=1}^{n} \int_{\Pi_{a, a+\varepsilon}} \varepsilon^{-1} \frac{\partial u_{k}}{\partial x_{i}} a_{i}^{(k)}(x) u_{k 0}(x) d x d t+\int_{\Pi_{a, a+\varepsilon}} \varepsilon^{-1} f_{k}(u, t) u_{k 0}(x, t) d x d t=0 .
\end{aligned}
$$

Устремив в этом неравенстве $\varepsilon$ к нулю, получим, что при почти всех $a>0$ имеет место равенство:

$$
\begin{aligned}
\int_{\substack{t=a \\
x \in \Omega}} u_{k 0}(x) \frac{\partial u_{k}}{\partial t} d x+\sum_{i, j=1}^{n} \int_{\substack{t=a \\
x \in \Omega}} a_{i j}^{(k)} \frac{\partial u_{k 0}}{\partial x_{i}} \frac{\partial u_{k}}{\partial x_{j}} d x \\
-\sum_{i=1}^{n} \int_{\substack{t=a \\
x \in \Omega}} \frac{\partial u_{k}}{\partial x_{i}} a_{i}^{(k)}(x) u_{k 0}(x) d x+\int_{\substack{t=a \\
x \in \Omega}} f_{k}(u, t) u_{k 0}(x) d x=0 .
\end{aligned}
$$

Так как $u_{k 0}(x)$ является обобщенным решением задачи $(18)$, то из $(21)$ получаем:

$$
\int_{x \in \Omega}^{t=a} u_{k 0}(x) \frac{\partial u_{k}}{\partial t} d x+\int_{\substack{t=a \\ x \in \Omega}} f_{k}(u, t) u_{k 0}(x) d x=0 .
$$

Будем обозначать:

$$
\bar{u}_{k}=(\operatorname{mes} \Omega)^{-1} \int_{\Omega} u_{k}(x, t) u_{k 0}(x) d x \text {. }
$$


Из (22) следует:

$$
\frac{d}{d t} \bar{u}_{k}+\overline{f_{k}(u, t)}=0, \quad t>0, \quad k=1, \ldots, N .
$$

Умножив обе части $(23)$ на $\psi_{k}(x, t) \in W_{2}^{1}\left(\Pi_{a, b}\right)$ и потом проинтегрировав по $\Pi_{a, b}$ получим:

$$
\begin{gathered}
\int_{\Pi_{a, b}} \psi_{k}(x, t) \frac{d}{d t} \overline{u_{k}(t)} d x d t+\sum_{i, j=1}^{n} \int_{\Pi_{a, b}} a_{i j}^{(k)}(x) \frac{\partial \psi_{k}}{\partial x_{i}} \frac{\partial \bar{u}_{k}}{\partial x_{j}} d x d t \\
-\sum_{i=1}^{n} \int_{\Pi_{a, b}} a_{i}^{(k)}(x) \frac{\partial \bar{u}_{k}}{\partial x_{i}} \psi_{k}(x, t) d x d t+\int_{\Pi_{a, b}} \overline{f_{k}(u(x, t), t)} \psi_{k}(x, t) d x d t=0,
\end{gathered}
$$

так как $\bar{u}_{k}$ не зависит от $x$. Из (17), (24) следует:

$$
\begin{gathered}
\int_{\Pi_{a, b}} \psi_{k} \frac{\partial}{\partial t}\left(u_{k}-\bar{u}_{k}\right) d x d t+\sum_{i, j=1}^{n} \int_{\Pi_{a, b}} a_{i j}^{(k)} \frac{\partial \psi_{k}}{\partial x_{i}} \frac{\partial\left(u_{k}-\bar{u}_{k}\right)}{\partial x_{j}} d x d t \\
-\sum_{i=1}^{n} \int_{\Pi_{a, b}} a_{i}^{(k)} \psi_{k} \frac{\partial}{\partial x_{i}}\left(u_{k}-\bar{u}_{k}\right) d x d t+\int_{\Pi_{a, b}}\left(f_{k}(u, t)-\overline{f_{k}(u, t)}\right) \psi_{k} d x d t=0
\end{gathered}
$$

если $\psi_{k} \in W_{2}^{1}\left(\Pi_{a, b}\right), k=1, \ldots, N$. Положим в $(25): \psi_{k}=\left(u_{k}-\bar{u}_{k}\right) u_{k 0}(x)$. В результате получим:

$$
\begin{aligned}
\sum_{k=1}^{N} \frac{1}{2} \int_{t=b} u_{k 0}\left(u_{k}-\bar{u}_{k}\right)^{2} d x-\sum_{k=1}^{N} \frac{1}{2} \int_{t=a} u_{k 0}(x)\left(u_{k}-\bar{u}_{k}\right)^{2} d x \\
+\sum_{k=1}^{N} \sum_{i, j=1}^{n} \int_{\Pi_{a, b}} a_{i j}^{(k)} u_{k 0} \frac{\partial\left(u_{k}-\bar{u}_{k}\right)}{\partial x_{i}} \frac{\partial\left(u_{k}-\bar{u}_{k}\right)}{\partial x_{j}} d x d t \\
+\sum_{k=1}^{N} \sum_{i, j=1}^{n} \frac{1}{2} \int_{\Pi_{a, b}} a_{i j}^{(k)} \frac{\partial u_{k 0}(x)}{\partial x_{i}} \frac{\partial\left(u_{k}-\bar{u}_{k}\right)^{2}}{\partial x_{j}} d x d t \\
\quad-\sum_{k=1}^{N} \sum_{i=1}^{n} \frac{1}{2} \int_{\Pi_{a, b}} a_{i}^{(k)} u_{k 0} \frac{\partial}{\partial x_{i}}\left(u_{k}-\bar{u}_{k}\right)^{2} d x d t \\
+\int_{\Pi_{a, b}} \sum_{k=1}^{N}\left(f_{k}(u, t)-\overline{f_{k}(u, t)}\right)\left(u_{k}-\bar{u}_{k}\right) u_{k 0}(x) d x d t=0
\end{aligned}
$$

Так как $u_{k 0}(x)$ есть обобшенное решение задачи (18), то равенство (26) сводится к следуюшему:

$$
\begin{aligned}
\sum_{k=1}^{N} \frac{1}{2} \int_{t=b} u_{k 0}(x)\left(u_{k}-\bar{u}_{k}\right)^{2} d x-\sum_{k=1}^{N} \frac{1}{2} \int_{t=a} u_{k 0}\left(u_{k}-\bar{u}_{k}\right)^{2} d x \\
+\sum_{k=1}^{N} \sum_{i, j=1}^{n} \int_{\Pi_{a, b}} a_{i j}^{(k)} u_{k 0} \frac{\partial\left(u_{k}-\bar{u}_{k}\right)}{\partial x_{i}} \frac{\partial\left(u_{k}-\bar{u}_{k}\right)}{\partial x_{j}} d x d t \\
\quad+\int_{\Pi_{a, b}} \sum_{k=1}^{N}\left(f_{k}(u, t)-\overline{f_{k}(u, t)}\right)\left(u_{k}-\bar{u}_{k}\right) u_{k 0} d x d t=0
\end{aligned}
$$


Из неравенства Пуанкаре следует:

$$
\begin{aligned}
\int_{\Omega}\left[f_{k}(u, t)-\overline{f_{k}(u, t)}\right]^{2} d x & \leqslant C \int_{\Omega} \sum_{i=1}^{n}\left[\frac{\partial}{\partial x_{i}}\left(f_{k}-\overline{f_{k}}\right)\right]^{2} d x \\
& \leqslant C_{1} \int_{\Omega}\left|\nabla_{u} f_{k}\right|^{2}|\nabla u|^{2} d x \leqslant \varepsilon \int_{\Omega}\left|\nabla_{x} u\right|^{2} d x
\end{aligned}
$$

если $t$ достаточно велико. Используя (15), (27), получаем:

$$
\sum_{k=1}^{N} \frac{1}{2} \int_{t=b} u_{k 0}\left(u_{k}-\bar{u}_{k}\right)^{2} d x-\sum_{k=1}^{N} \frac{1}{2} \int_{t=a} u_{k 0}\left(u_{k}-\bar{u}_{k}\right)^{2} d x+c \int_{\Pi_{a, b}}\left|\nabla_{x} u\right|^{2} d x d t \leqslant 0 .
$$

Устремив $b \mathrm{k}+\infty$, получим:

$$
-\sum_{k=1}^{N} \frac{1}{2} \int_{t=a} u_{k 0}\left(u_{k}-\bar{u}_{k}\right)^{2} d x+c \int_{\Pi_{a}}\left|\nabla_{x} u\right|^{2} d x d t \leqslant 0
$$

Используя неравенство Пуанкаре, имеем

$$
\int_{\Pi_{a}}\left|\nabla_{x} u\right|^{2} d x d t \leqslant c_{1} \int_{t=a}\left|\nabla_{x} u\right|^{2} d x
$$
Положим $J(\tau)=\int_{\Pi_{\tau}}\left|\nabla_{x} u\right|^{2} d x$. Теперь (28) можно переписать следующим обра-
зом

$$
J(\tau) \leqslant-c_{1} \frac{d J}{d \tau}
$$

Интегрируя это неравенство, получим

$$
J(\tau) \leqslant C e^{-\alpha \tau}, \quad \alpha=c_{1}^{-1} .
$$

Из (29) и из неравенства Пуанкаре следует:

$$
\int_{\Pi_{a}}\left|\nabla_{x} u\right|^{2} d x d t+\int_{\Pi_{a}} \sum_{k=1}^{N}\left|u_{k}-\bar{u}_{k}\right|^{2} d x d t \leqslant c_{2} e^{-\alpha a} .
$$

Перепишем систему уравнений (23) в виде:

$$
\frac{d}{d t} \bar{u}_{s}+f_{s}(\bar{u}, t)=g_{s}(t), \quad s=1, \ldots, N, \quad t>0
$$

где $g_{s}=f_{s}(\bar{u}, t)-\overline{f_{s}(u, t)}$. Легко видеть, что

$$
g_{s}=f_{s}(\bar{u}, t)-\overline{f_{s}(u, t)}+\overline{f_{s}(\bar{u}, t)}-\overline{f_{s}(\bar{u}, t)}=\overline{f_{s}(\bar{u}, t)-f_{s}(u, t)} .
$$

Так как $\left|f_{s}(u, t)-f_{s}(\bar{u}, t)\right| \leqslant\left|\nabla_{u} f_{s} \| u-\bar{u}\right|$ и $u(x)$ ограничена, то

$$
\left|g_{s}(t)\right| \leqslant c_{2} \int_{\Omega} \sum_{k=1}^{N}|u-\bar{u}| d x .
$$


Отсюда

$$
\left|g_{s}\right|^{2} \leqslant c_{3} \int_{\Omega} \sum_{k=1}^{N}|u-\bar{u}|^{2} d x
$$

Из (30) следует

$$
\int_{\Pi_{t}} g_{s}^{2} d x d t \leqslant c_{3} e^{-\alpha t}
$$

К системе (31) можно применить теорему 1 и получить, что

$$
\bar{u}=X(t)+v_{1}(t)
$$

где $\left\|v_{1}\right\| \equiv O\left(e^{-\alpha t}\right)$.

Отсюда и из (30) следует (20).

Теорема доказана.

Рассмотрим случай одного уравнения $(N=1)$. В этом случае имеет место более сильный результат.

ТЕОРЕма 3. Если и $(x, t)$ - решение системы $(14)$, где $N=1$, удовлетворяет условию (16) и

$$
\lim _{t \rightarrow \infty} u(x, t)=0
$$

mo

$$
u(x, t)=\chi(t)+v(x, t),
$$

где $\chi(t)$ - некоторое решение уравнения (19), а

$$
v(x, t)=O\left(e^{-\alpha t}\right), \quad \alpha=\text { const }>0 .
$$

ДокАЗАТЕЛьство. Из теоремы 2 следует, что

$$
u(x, t)=\chi(t)+v(x, t),
$$

где $v(x, t)$ удовлетворяет неравенству (20). Подставим выражение (33) в (14). В результате получим:

$$
\frac{\partial v}{\partial t}=\sum_{i, j=1}^{n} \frac{\partial}{\partial x_{i}} a_{i j}(x) \frac{\partial v}{\partial x_{j}}+\sum_{i=1}^{n} a_{i}(x) \frac{\partial v}{\partial x_{i}}+f(\chi+v, t)-f(\chi, t) .
$$

Заметим, что $f(\chi+v, t)-f(\chi, t)=v P(x, t)$, где $P(x, t)$ - ограниченная функция.

Уравнение (34) можно переписать в виде:

$$
\frac{\partial v}{\partial t}=\sum_{i, j=1}^{n} \frac{\partial}{\partial x_{i}} a_{i j}(x) \frac{\partial v}{\partial x_{j}}+\sum_{i=1}^{n} a_{i}(x) \frac{\partial v}{\partial x_{i}}+P(x, t) v .
$$

Из (20) и из оценок типа Нэша [3] следует (32). Теорема доказана.

Отметим, что утверждение теоремы 3 остается верным и для системы уравнений (14) при любом $N$, если коэффициенты $a_{i j}^{(k)}(x)$ - гладкие функции, а $\partial \Omega$ - гладкая поверхность.

Приведем несколько примеров применения теорем 2,3 . 
1. Рассмотрим уравнение

$$
\begin{gathered}
\frac{\partial u}{\partial t}=\sum_{i, j=1}^{n} \frac{\partial}{\partial x_{i}} a_{i j}(x) \frac{\partial u}{\partial x_{j}}+\sum_{i=1}^{n} a_{i}(x) \frac{\partial u}{\partial x_{i}}-a_{0}|u|^{\sigma-1} u, \\
\sigma=\text { const }>1, \quad a_{0}=\text { const }>0, \quad(x, t) \in \Pi_{0},
\end{gathered}
$$

и его решение $u(x, t)$, удовлетворяюшее условию:

$$
\left.\frac{\partial u}{\partial \nu}\right|_{\partial \Omega}=0, \quad t>0
$$

где $\nu$ - вектор внешней конормали.

Уравнение (35) предполагается параболическим, а его коэффициенты - ограниченные измеримые функции. Из принципа максимума следует, что

$$
\lim _{t \rightarrow+\infty} u=0
$$

для любого решения уравнения (35), которое удовлетворяет условию (36). Применив теорему 3 , получаем, что всякое решение задачи $(35),(36)$ имеет вид: либо

$$
u= \pm c_{\sigma}(t+T)^{\frac{1}{1-\sigma}}+O\left(e^{-\alpha t}\right)
$$

где $\frac{1}{a_{0}(\sigma-1)}=c_{\sigma}^{\sigma-1}$, а постоянная $T$ зависит от $u$, либо

$$
u=O\left(e^{-\alpha t}\right) .
$$

Случай (37) имеет место тогда и только тогда, когда решение $u(x, t)$ меняет знак в каждой области $\Pi_{t}, t>0$, ибо в противном случае, в силу принципа максимума

$$
|u| \geqslant c_{\sigma}(t+T)^{\frac{1}{1-\sigma}}
$$

2. Рассмотрим в $\Pi_{0}$ уравнение

$$
\frac{\partial u}{\partial t}=\Delta u-u(\ln |u|+1)^{-s}, \quad s>0 .
$$

Легко видеть, что каждое его решение, удовлетворяюшее краевому условию Неймана на $\sigma_{0}$, имеет нулевой предел при $t \rightarrow+\infty$ и

$$
u(x, t)= \pm e^{-1} e^{-(s+1)(t+T)^{\frac{1}{s+1}}}+O\left(e^{-\alpha t}\right), \quad \alpha>0
$$

или $u=O\left(e^{-\alpha t}\right)$. Второй случай имеет место тогда и только тогда, когда $u$ меняет знак в каждой области $\Pi_{t}, t>0$. 
3. Рассмотрим уравнение:

$$
\frac{\partial u}{\partial t}=\sum_{i, j=1}^{n} \frac{\partial}{\partial x_{i}} a_{i j}(x) \frac{\partial u}{\partial x_{j}}+\sum_{i=1}^{n} a_{i}(x) \frac{\partial u}{\partial x_{i}}-\frac{|u|^{\sigma-1} u}{(1+t)^{s}}
$$

где $\sigma>1, s \leqslant 1,(x, t) \in \Pi_{0}$.

Всякое его решение, удовлетворяюшее краевому условию (30), имеет следующую асимптотику при $t \rightarrow+\infty$ :

при $s<1$ либо

$$
u= \pm\left(\frac{\sigma-1}{1-s} t^{1-s}+T\right)^{\frac{1}{1-\sigma}}+O\left(e^{-\alpha t}\right)
$$

либо $u=O\left(e^{-\alpha t}\right)$.

При $s=1$ или

$$
u= \pm[(\sigma-1) \ln t T]^{\frac{1}{1-\sigma}}+O\left(e^{-\alpha t}\right)
$$

или $u=O\left(e^{-\alpha t}\right)$.

В обоих случаях асимптотика $u=O\left(e^{-\alpha t}\right)$ имеет место тогда и только тогда, когда $u(x, t)$ меняет знак в любой области $\Pi_{a}, a>0$.

4. Пусть уравнение имеет вид:

$$
\frac{\partial u}{\partial t}=\Delta u+|u|^{\sigma-1} u, \quad \sigma>1
$$

Пусть $u(x, t)$ - его решение в $\Pi_{0}$ - удовлетворяет краевому условию $(36)$ и таково, что $\lim _{t \rightarrow \infty} u(x, t)=0$.

Тогда

$$
u=O\left(e^{-\alpha t}\right), \quad \alpha>0 .
$$

Известно, что не сушествует положительных решений уравнения (39) в $\Pi_{0}$, которые удовлетворяют (36). Можно доказать существование знакопеременных решений в $\Pi_{0}$, для них имеет место (40).

5. Рассмотрим уравнение (38) при $s>1$. В этом случае всякое решение, которое удовлетворяет условию (36), имеет конечньй предел при $t \rightarrow+\infty$. Нулевой предел может иметь только то решение, которое меняет знак в каждой области $\Pi_{a}, a>0$. Для него справедлива формула (40). Если решение знакопостоянно в некоторой области $\Pi_{a}$, то $\lim _{t \rightarrow+\infty} u(x, t)=c \neq 0$ и

$$
u=\left[c+\frac{1-\sigma}{1-s}(t+1)^{-s+1}\right]^{\frac{1}{1-\sigma}}+O\left(e^{-\alpha t}\right) .
$$

6. Рассмотрим уравнение:

$$
\frac{\partial u}{\partial t}=\Delta u+u-k|u|^{\sigma-1} u, \quad(x, t) \in \Pi_{0},
$$

где $\sigma=$ const $>0, k=$ const, $\partial \Omega$ - липшицева поверхность. 
ТЕОРема 4. Если и $(x, t)$ - решение уравнения (41) в П у удовлетворяет краевому условию

$$
\frac{\partial u}{\partial n}=0, \quad x \in \partial \Omega, \quad t>0
$$

$u \lim _{t \rightarrow \infty} u(x, t)=0$, а область $\Omega$ такова, что наименьшее ненулевое собственное значение задачи Неймана для оператора Лапласа по модулю больше 1 , то справедлива формула (40).

ДокАЗАтЕЛьство. Будем обозначать $\bar{u}=(\operatorname{mes} \Omega)^{-1} \int_{\Omega} u d x$.

Из (41) следует

$$
\frac{\partial \bar{u}}{\partial t}=\bar{u}-k \overline{|u|^{\sigma-1} u}
$$

Следовательно,

$$
\frac{\partial(u-\bar{u})}{\partial t}=\Delta(u-\bar{u})+(u-\bar{u})+k\left[|u|^{\sigma-1} u-\overline{|u|^{\sigma-1}} u\right] .
$$

Отсюда

$$
\begin{aligned}
\int_{\substack{t=b \\
x \in \Omega}} & \frac{(u-\bar{u})^{2}}{2} d x-\int_{\substack{t=a \\
x \in \Omega}} \frac{(u-\bar{u})^{2}}{2} d x+\int_{\Pi_{a, b}}\left|\nabla_{x}(u-\bar{u})\right|^{2} d x d t \\
& -\int_{\Pi_{a, b}}(u-\bar{u})^{2} d x d t+k \int_{\Pi_{a, b}}(u-\bar{u})\left[|u|^{\sigma-1} u-\overline{|u|^{\sigma-1} u}\right] d x d t=0 .
\end{aligned}
$$

Оценим последний интеграл в левой части этой формулы:

$$
\begin{aligned}
& \left|\int_{\Pi_{a, b}}(u-\bar{u})\left[|u|^{\sigma-1} u-\overline{|u|^{\sigma-1} u}\right] d x d t\right| \\
& \quad \leqslant\left(\int_{\Pi a, b}|u-\bar{u}|^{2} d x d t\right)^{1 / 2}\left(\left.\int_{\Pi_{a, b}}|| u\right|^{\sigma-1} u-\left.\overline{|u|^{\sigma-1} u}\right|^{2} d x d t\right)^{1 / 2}
\end{aligned}
$$

Используя теорему Лагранжа и неравенство Пуанкаре, получаем:

$$
\left|\int_{\Pi_{a, b}}(u-\bar{u})\left[\left.|| u\right|^{\sigma-1} u-\overline{|u|^{\sigma-1} u} \mid\right] d x d t\right| \leqslant \varepsilon \int_{\Pi_{a, b}}\left|\nabla_{x} u\right|^{2} d x d t
$$

если $а$ достаточно велико. Поэтому из (42) следует:

$$
\begin{aligned}
\int_{\substack{t=b \\
x \in \Omega}} \frac{|u-\bar{u}|^{2}}{2} d x & -\int_{\substack{t=a \\
x \in \Omega}} \frac{|u-\bar{u}|^{2}}{2} d x \\
& +(1-\varepsilon) \int_{\Pi_{a, b}}\left|\nabla_{x}(u-\bar{u})\right|^{2} d x d t-\int_{\Pi_{a, b}}|u-\bar{u}|^{2} d x d t \leqslant 0 .
\end{aligned}
$$

Используя неравенство Пуанкаре

$$
\int_{\Omega}(u-\bar{u})^{2} d x \leqslant\left|\lambda_{2}\right|^{-1} \int_{\Omega}\left(\nabla_{x} u\right)^{2} d x
$$


получаем

$$
\frac{\varepsilon}{2} \int_{\Pi_{a, b}}\left|\nabla_{x}(u-\bar{u})\right|^{2} d x d t \leqslant-\int_{\substack{t=b \\ x \in \Omega}} \frac{|u-\bar{u}|^{2}}{2} d x+\int_{\substack{t=a \\ x \in \Omega}} \frac{|u-\bar{u}|^{2}}{2} d x .
$$

Устремив $b \mathrm{~K}+\infty$, получим

$$
\int_{\Pi_{a}}\left|\nabla_{x}(u-\bar{u})\right|^{2} d x d t \leqslant \int_{\substack{t=a \\ x \in \Omega}}\left|\nabla_{x}(u-\bar{u})\right|^{2} d x
$$

Отсюда следует (это рассуждение было подробно проведено выше при доказательстве теоремы 2), что

$$
\int_{\Pi_{\tau}}\left|\nabla_{x}(u-\bar{u})\right|^{2} d x \leqslant c e^{-2 \alpha \tau} .
$$

Применяя еще раз неравенство Пуанкаре, получаем

$$
\int_{\Pi_{\tau}}|u-\bar{u}|^{2} d x \leqslant c_{1} e^{-2 \alpha \tau}
$$

Перепишем уравнение (41) в виде:

$$
\frac{d \bar{u}}{d t}=\bar{u}-k \overline{|u|^{\sigma-1} u}+k|\bar{u}|^{\sigma-1} \bar{u}-k|\bar{u}|^{\sigma-1} \bar{u}=\bar{u}-k|\bar{u}|^{\sigma-1} \bar{u}+F(t),
$$

где

$$
\begin{aligned}
F(t) & =k|\bar{u}|^{\sigma-1} \bar{u}-k \overline{|u|^{\sigma-1} u}=k \overline{\left.\bar{u}\right|^{\sigma-1} \bar{u}}-k \overline{|u|^{\sigma-1} u} \\
& =k(\operatorname{mes} \Omega)^{-1} \int_{\Omega}\left[|\bar{u}|^{\sigma-1} \bar{u}-|u|^{\sigma-1} u\right] d x \leqslant c_{2} \int_{\Omega}|u-\bar{u}| d x
\end{aligned}
$$

Отсюда

$$
\int_{t}^{\infty} F^{2}(t) d t \leqslant c_{2} \int_{\Pi_{t}}|u-\bar{u}|^{2} d x \leqslant c_{3} e^{-2 \alpha \tau} .
$$

Из (44) и из теоремы 1 следует:

$$
\bar{u}=\chi(t)+v(t)
$$

где $\chi(t)$ - некоторое решение уравнения:

$$
\frac{d X}{d t}=X-k|X|^{\sigma-1} X
$$

имеющее нулевой предел при $t \rightarrow+\infty$, a

$$
v(t)=O\left(e^{-\alpha t}\right) .
$$

Из (45) следует, что $X(t) \equiv 0$.

Таким образом,

$$
\bar{u}=O\left(e^{-\alpha t}\right) .
$$


Отсюда и из (43)

$$
\int_{\Pi_{\tau}}|u|^{2} d x \leqslant c_{4} e^{-2 \alpha \tau}
$$

Используя неравенство Нэша [3], получаем

$$
|u(x, t)| \leqslant c_{5} e^{-\alpha t}
$$

что и требуется. Теорема 4 доказана.

Перейдем к рассмотрению эллиптических уравнений и систем уравнений.

Рассмотрим систему уравнений:

$$
\begin{gathered}
L_{k} u \equiv \sum_{i, j=1}^{n} \frac{\partial}{\partial x_{i}} a_{i j}^{(k)}(\widehat{x}) \frac{\partial u_{k}}{\partial x_{j}}+\sum_{i=1}^{n} a_{i}^{(k)}(\widehat{x}) \frac{\partial u_{k}}{\partial x_{i}}=f_{k}\left(u, x_{n}\right) \\
k=1, \ldots, N, \quad u=\left(u_{1}, \ldots, u_{N}\right)
\end{gathered}
$$

Будем предполагать, что все коэффициенты в (47) - ограниченные измеримые функции, $x=\left(\widehat{x}, x_{n}\right), \widehat{x}=\left(x_{1}, \ldots, x_{n-1}\right)$. Уравнение $(47)$ рассматривается в цилиндрической области $\Pi_{0}=\left\{x: \widehat{x} \in \Omega, 0<x_{n}<\infty\right\}$, где $\Omega$ - ограниченная область с липшицевой границей. Предполагается выполненньм условие эллиптичности:

$$
m_{1}|\xi|^{2} \leqslant \sum_{i, j=1}^{n} a_{i j}^{(k)}(\widehat{x}) \xi_{i} \xi_{j} \leqslant m_{2}|\xi|^{2}, \quad k=1, \ldots, N
$$

где

$$
m_{1}, m_{2}=\mathrm{const}, \quad m_{1}>0, \quad|\xi|^{2}=\sum_{i=1}^{n} \xi_{i}^{2}, \quad u=\left(u_{1}, \ldots, u_{N}\right) \text {. }
$$

Пусть, кроме того, $a_{n n}^{(k)} \equiv 1, a_{i n}^{(k)} \equiv 0$ при $i<n, a_{i j}^{(k)} \equiv a_{j i}^{(k)}, a_{n}^{(k)} \equiv$ const. Функции $f_{k}\left(u, x_{n}\right)$ определены при $0 \leqslant x_{n}<+\infty,\|u\| \leqslant a\left(\|u\|=\max _{i \leqslant N}\left(u_{i}\right)\right)$, где они непрерывны по $u$ и измеримы по $x_{n}$. Предполагаем, что $f(0, t) \equiv 0$ и существуют производные $\frac{\partial f_{k}}{\partial u_{j}}, j, k=1, \ldots, N$, причем

$$
\lim _{u \rightarrow 0} \frac{\partial f_{k}}{\partial u_{j}}=0, \quad k, j=1, \ldots, N
$$

равномерно по $x_{n}$.

Будем рассматривать решение системы уравнений (47) в $\Pi_{0}$, предполагая выполненным краевое условие:

$$
\frac{\partial u_{i}}{\partial \nu_{i}}=0, \quad \widehat{x} \in \partial \Omega, \quad x_{n}>0, \quad i=1, \ldots, N
$$

где $\frac{\partial}{\partial \nu_{i}}-$ дифференцирование по направлению конормали оператора $L_{i}$. В качестве решения задачи $(47),(49)$ понимается обобшенное решение. Приведем его определение. Будем обозначать $\Pi_{a, b}=\Omega \times(a, b), \Pi_{a, \infty}=\Pi_{a}, \Gamma_{a, b}=\partial \Omega \times(a, b)$, 
$\Gamma_{a, \infty}=\Gamma_{a}$. Вектор-функция $u(x)=\left(u_{1}, \ldots, u_{N}\right)$ называется обобщенным решением уравнения (47), удовлетворяюшим условию (49), если $u_{i} \in W_{2}^{1}\left(\Pi_{a, b}\right)$, $i=1, \ldots, N$, при любых $a, b<\infty,\|u\| \leqslant a$, и имеет место равенство

$$
\begin{aligned}
-\int_{\Pi_{a, b}} \sum_{k=1}^{N} \sum_{i, j=1}^{n} a_{i j}^{(k)} \frac{\partial \psi_{k}}{\partial x_{i}} \frac{\partial u_{k}}{\partial x_{j}} d x \\
\quad+\int_{\Pi_{a, b}} \sum_{k=1}^{N} \sum_{i=1}^{n} a_{i}^{(k)} \psi_{k} \frac{\partial u_{k}}{\partial x_{i}} d x-\sum_{k=1}^{N} \int_{\Pi_{a, b}} \psi_{k} f_{k}\left(u, x_{n}\right) d x=0
\end{aligned}
$$

каковы бы ни были функции $\psi_{k}(x) \in W_{2}^{1}\left(\Pi_{a, b}\right)$ такие, что $\left.\psi_{k}\right|_{x_{n}=0}=\left.\psi_{k}\right|_{x_{n}=b}=0$.

Из классических результатов о гладкости обобщенных решений эллиптических уравнений следует, что $u_{i}(x), i=1, \ldots, N$, непрерывны в $\bar{\Pi}_{a}$ при всех $a>0$ и в каждой замкнутой области $\bar{\Pi}_{a, b}$ удовлетворяют условию Гёльдера, $0<a<$ $b<\infty$.

Кроме того, $\frac{\partial u_{i}}{\partial x_{n}} \in W_{2}^{1}\left(\Pi_{a, b}\right), 0<a<b<\infty, i=1, \ldots, N$.

Как и при исследовании решений параболических систем, будут использоваться функции $u_{k 0}(\widehat{x})$ - нетривиальные решения систем:

$$
\sum_{i, j=1}^{n-1} \frac{\partial}{\partial x_{i}} a_{i j}^{(k)}(\widehat{x}) \frac{\partial u_{k 0}}{\partial x_{j}}-\sum_{i=1}^{n-1} \frac{\partial}{\partial x_{i}} a_{i}^{(k)}(\widehat{x}) u_{k 0}=0, \quad k=1, \ldots, N
$$

с краевыми условиями:

$$
\frac{\partial u_{k 0}}{\partial \nu_{k}}-\sum_{i=1}^{n} a_{i}^{(k)}(\widehat{x}) u_{k 0} \cos (n, x)=0
$$

Функции $u_{k 0}(\widehat{x})$ непрерывны в $\bar{\Omega}$ и не обрашаются в нуль в $\bar{\Omega}$. Будем считать, что $u_{k 0}(\widehat{x})>0$ и

$$
\int_{\Omega} u_{k 0}(\widehat{x}) d \widehat{x}=1
$$

Teорема 5. Существует $\rho_{0}=$ const $>0$ такое, что если $и(x)-$ решение задачи (47), (49) таково, что $\|u(x)\| \leqslant \rho, x \in \Pi_{a}$, при некотором а $>0$, то

$$
u(x)=\chi\left(x_{n}\right)+v(x)
$$

где $\chi(t)$ - решение системь

$$
\ddot{x}_{k}+a_{n}^{(k)} \dot{x}_{k}=f_{k}(x, t), \quad k=1, \ldots, N, \quad x=\left(x_{1}, \ldots, x_{n}\right),
$$

$a$

$$
\int_{\Pi_{T}} v^{2} d x=O\left(e^{-2 \alpha T}\right), \quad \alpha=\text { const }>0
$$


ДокАЗАТЕЛЬСТво. Пусть $\Theta\left(x_{n}\right) \in C^{\infty}\left(\mathbb{R}^{1}\right), \Theta\left(x_{n}\right) \equiv 0$ при $x_{n}<a$ и при $x_{n}>a+\varepsilon, \Theta\left(x_{n}\right) \geqslant 0, \int_{-\infty}^{+\infty} \Theta\left(x_{n}\right) d x_{n}=1$. Зафиксируем $k, 1 \leqslant k \leqslant N$, и в равенстве (50) положим: $\psi_{k}=\Theta\left(x_{n}\right) u_{k 0}(\widehat{x}), \psi_{i} \equiv 0$ при $i \neq k$.

В результате получим, после интегрирования по частям:

$$
\begin{aligned}
& \int_{\Pi_{a, a+\varepsilon}} \Theta\left(x_{n}\right) u_{k 0}(\widehat{x}) \frac{\partial^{2} u}{\partial x_{n}^{2}} d x-\sum_{i, j=1}^{n-1} \int_{\Pi_{a, a+\varepsilon}} a_{i j}(\widehat{x}) \Theta\left(x_{n}\right) \frac{\partial u_{k 0}}{\partial x_{i}} \frac{\partial u_{k}}{\partial x_{j}} d x \\
& +\sum_{i=1}^{n} \int_{\Pi_{a, a+\varepsilon}} u_{k 0}(\widehat{x}) a_{i}(\widehat{x}) \Theta\left(x_{n}\right) \frac{\partial u_{k}}{\partial x_{i}} d x-\int_{\Pi_{a, a+\varepsilon}} \Theta\left(x_{n}\right) u_{k 0}(\widehat{x}) f_{k}\left(u(x), x_{n}\right) d x=0 .
\end{aligned}
$$

Устремив $\varepsilon$ к нулю, получим, что при почти всех $a>0$ :

$$
\begin{aligned}
\int_{\substack{x_{n}=a \\
\widehat{x} \in \Omega}} u_{k 0}(\widehat{x}) & \frac{\partial^{2} u_{k}}{\partial x_{n}^{2}} d \widehat{x}-\sum_{i, j=1}^{n-1} \int_{\substack{x_{n}=a \\
\widehat{x} \in \Omega}} a_{i j}(\widehat{x}) \frac{\partial u_{k 0}}{\partial x_{i}} \frac{\partial u_{k}}{\partial x_{j}} d \widehat{x} \\
& +\sum_{i=1}^{n} \int_{\substack{x_{n}=a \\
\widehat{x} \in \Omega}} u_{k 0}(\widehat{x}) a_{i}(x) \frac{\partial u_{k}}{\partial x_{i}} d \widehat{x}-\int_{\substack{x_{n}=a \\
\widehat{x} \in \Omega}} u_{k 0}(\widehat{x}) f_{k}(x, u) d \widehat{x}=0 .
\end{aligned}
$$

Из определения $u_{k 0}(\widehat{x})$ следует:

$$
\begin{aligned}
& \frac{\partial^{2}}{\partial x_{n}^{2}} \int_{\substack{x_{n}=a \\
\widehat{x} \in \Omega}} u_{k 0}(\widehat{x}) u_{k}(x) d \widehat{x} \\
& \quad+\int_{\substack{x_{n}=a \\
\widehat{x} \in \Omega}} a_{n} \frac{\partial}{\partial x_{n}} u_{k 0}(\widehat{x}) u_{k}(x) d \widehat{x}-\int_{\substack{x_{n}=0 \\
\widehat{x} \in \Omega}} u_{k 0}(\widehat{x}) f_{k}(x, u(x)) d \widehat{x}=0 .
\end{aligned}
$$

Будем обозначать $\overline{g_{k}(x)}=\int_{\Omega} g_{k}(x) u_{k 0}(\widehat{x}) d \widehat{x}$. Теперь формулу (53) можно переписать в виде:

$$
\frac{d^{2}}{d x_{n}^{2}} \bar{u}_{k}+a_{n}^{(k)} \frac{d}{d x_{n}} \bar{u}_{k}=\overline{f_{k}\left(x_{n}, u\right)}, \quad k=1, \ldots, N, \quad x_{n}>0 .
$$

Из (54) следует, что каковы бы ни были функции $\psi_{k}(x), k=1, \ldots, N$, такие, что $\psi_{k} \in W_{2}^{1}(a, b),\left.\psi_{k}\right|_{x_{n}=a}=\left.\psi_{k}\right|_{x_{n}=b}=0, k=1, \ldots, N$, имеет место тождество:

$$
-\int_{\Pi_{a, b}} \frac{\partial \psi_{k}(x)}{\partial x_{n}} \frac{\partial}{\partial x_{n}} \bar{u}_{k} d x+a_{n}^{(k)} \int_{\Pi_{a, b}} \psi_{k}(x) \frac{\partial}{\partial x_{n}} \bar{u}_{k} d x=\int_{\Pi_{a, b}} \psi_{k} \overline{f_{k}\left(x_{n}, u\right)} d x
$$

Из (50), (55) следует:

$$
\begin{array}{r}
-\int_{\Pi_{a, b}} \sum_{i, j=1}^{n} \sum_{k=1}^{N} a_{i j}^{(k)} \frac{\partial}{\partial x_{i}} \psi_{k}(x) \frac{\partial}{\partial x_{j}}\left(u_{k}-\bar{u}_{k}\right) d x+\sum_{k=1}^{N} \sum_{i=1}^{n} \int_{\Pi_{a, b}} a_{i}^{k} \psi_{k} \frac{\partial}{\partial x_{i}}\left(u_{k}-\bar{u}_{k}\right) d x \\
=\sum_{k=1}^{N} \int_{\Pi_{a, b}} \psi_{k}\left(\overline{f_{k}\left(u, x_{n}\right)}-f_{k}\left(u, x_{n}\right)\right) d x,
\end{array}
$$


если $\psi_{k} \in W_{2}^{1}\left(\Pi_{a, b}\right),\left.\psi\right|_{x_{n}=a}=\left.\psi\right|_{x_{n}=b}=0$. Пусть $\Theta\left(x_{n}\right)$ - непрерывная на $(-\infty,+\infty)$ функция такая, что $\Theta\left(x_{n}\right) \equiv 0$ при $x_{n}<a, \Theta\left(x_{n}\right) \equiv 0$ при $x_{n}>b$, $\Theta\left(x_{n}\right) \equiv 1$, если $a+\varepsilon<x_{n}<b-\varepsilon$, и $\Theta\left(x_{n}\right)$ линейна на $(a, a+\varepsilon)$ и $(b-\varepsilon, b)$. Положим в $(56) \psi_{k}=\Theta\left(x_{n}\right) u_{k 0}(\widehat{x})\left(u_{k}-\bar{u}_{k}\right)$. В результате получим:

$$
\begin{gathered}
-\int_{\Pi_{a, b}} \sum_{i, j=1}^{n} \sum_{k=1}^{N} a_{i j}^{(k)}(\widehat{x}) \Theta\left(x_{n}\right) u_{k 0}(\widehat{x}) \frac{\partial}{\partial x_{i}}\left(u_{k}-\bar{u}_{k}\right) \frac{\partial}{\partial x_{j}}\left(u_{k}-\bar{u}_{k}\right) d x \\
-\int_{\Pi_{a, b}} \Theta\left(x_{n}\right) \sum_{i, j=1}^{n-1} \sum_{k=1}^{N} a_{i j}^{(k)}(\widehat{x})\left(u_{k}-\bar{u}_{k}\right)\left(\frac{\partial}{\partial x_{i}} u_{k 0}(\widehat{x})\right) \frac{\partial}{\partial x_{j}}\left(u_{k}-\bar{u}_{k}\right) d x \\
-\int_{\Pi_{a, b}} \sum_{k=1}^{N} u_{k 0}(\widehat{x}) \Theta^{\prime}\left(x_{n}\right)\left(u_{k}-\bar{u}_{k}\right) \frac{\partial}{\partial x_{n}}\left(u_{k}-\bar{u}_{k}\right) d x \\
+\int_{\Pi a, b} \sum_{k=1}^{N} \sum_{i=1}^{n-1} a_{i}^{(k)}(\widehat{x}) \Theta\left(x_{n}\right) u_{k 0}(\widehat{x})\left(u_{k}-\bar{u}_{k}\right) \frac{\partial}{\partial x_{i}}\left(u_{k}-\bar{u}_{k}\right) d x \\
+\int_{\Pi_{a, b}} \frac{1}{2} \sum_{k=1}^{N} a_{n}^{(k)} \Theta\left(x_{n}\right) u_{k 0}(\widehat{x}) \frac{\partial}{\partial x_{n}}\left(u_{k}-\bar{u}_{k}\right)^{2} d x \\
-\sum_{k=1}^{N} \int_{\Pi_{a, b}} \Theta\left(x_{n}\right) u_{k 0}(\widehat{x})\left(u_{k}-\bar{u}_{k}\right)\left(\bar{f}_{k}-f_{k}\right) d x=0 .
\end{gathered}
$$

Устремим $\varepsilon$ к нулю. В результате получим, что при почти всех $a, b$

$$
\begin{aligned}
-\int_{\Pi_{a}, b} \sum_{i, j=1}^{n} \sum_{k=1}^{N} a_{i j}^{(k)} u_{k 0}(\widehat{x}) \frac{\partial}{\partial x_{i}}\left(u_{k}-\bar{u}_{k}\right) \frac{\partial}{\partial x_{j}}\left(u_{k}-\bar{u}_{k}\right) & \\
& +\int_{\widehat{x} \in \Omega}=b \sum_{k=1}^{N} u_{k 0}(\widehat{x})\left(u_{k}-\bar{u}_{k}\right) \frac{\partial}{\partial x_{n}}\left(u_{k}-\bar{u}_{k}\right) \\
& -\int_{\widehat{x} \in \Omega} \sum_{k=1}^{N} u_{k 0}(\widehat{x})\left(u_{k}-\bar{u}_{k}\right) \frac{\partial}{\partial x_{n}}\left(u_{k}-\bar{u}_{k}\right) d \widehat{x} \\
& -\int_{\Pi_{a, b}} \frac{1}{2} \sum_{i, j=1}^{n-1} \sum_{k=1}^{N} a_{i j}^{(k)}(\widehat{x}) \frac{\partial u_{k 0}(\widehat{x})}{\partial x_{i}} \frac{\partial\left(u_{k}-\bar{u}_{k}\right)^{2}}{\partial x_{j}} \\
+ & \int_{\Pi_{a, b}} \frac{1}{2} \sum_{k=1}^{N} \sum_{i=1}^{n-1} a_{i}^{(k)}(\widehat{x}) u_{k 0}(\widehat{x}) \frac{\partial}{\partial x_{i}}\left(u_{k}-\bar{u}_{k}\right)^{2} d x \\
& +\frac{1}{2} \int_{\Pi_{a, b}} \sum_{k=1}^{N} a_{n}^{(k)} u_{k 0}(\widehat{x}) \frac{\partial}{\partial x_{n}}\left(u_{k}-\bar{u}_{k}\right)^{2} d x \\
& -\sum_{k=1}^{N} \int_{\Pi_{a, b}} u_{k 0}(\widehat{x})\left(u_{k}-\bar{u}_{k}\right)\left(\bar{f}_{k}-f_{k}\right) d x=0 .
\end{aligned}
$$


Так как $u_{k 0}(\widehat{x})$ - решение системы $(51)$, то из (57) получаем:

$$
\begin{aligned}
-\int_{\Pi_{a, b}} \sum_{i, j=1}^{n} \sum_{k=1}^{N} a_{i j}^{(k)} u_{k 0}(\widehat{x}) \frac{\partial}{\partial x_{i}}\left(u_{k}-\bar{u}_{k}\right) \frac{\partial}{\partial x_{j}}\left(u_{k}-\bar{u}_{k}\right) d x \\
+\int_{\widehat{x},=b} \sum_{k=1}^{N} u_{k 0}(\widehat{x})\left(u_{k}-\bar{u}_{k}\right) \frac{\partial}{\partial x_{n}}\left(u_{k}-\bar{u}_{k}\right) d \widehat{x} \\
-\int_{\widehat{x} \in \Omega} \sum_{k=1}^{N} u_{k 0}(\widehat{x})\left(u_{k}-\bar{u}_{k}\right) \frac{\partial}{\partial x_{n}}\left(u_{k}-\bar{u}_{k}\right) d \widehat{x} \\
\quad+\frac{1}{2} \int_{\widehat{x} \in \Omega}^{x_{n}=b} \sum_{k=1}^{N} a_{n}^{(k)} u_{k 0}(\widehat{x})\left(u_{k}-\bar{u}_{k}\right)^{2} d \widehat{x} \\
\quad-\frac{1}{2} \int_{\widehat{x} \in \Omega}^{x_{n}=a} \sum_{k=1}^{N} a_{n}^{(k)} u_{k 0}(\widehat{x})\left(u_{k}-\bar{u}_{k}\right)^{2} d \widehat{x} \\
\quad-\sum_{k=1}^{N} \int_{\Pi_{a, b}} u_{k 0}(\widehat{x})\left(u_{k}-\bar{u}_{k}\right)\left(\bar{f}_{k}-f_{k}\right) d \widehat{x}=0 .
\end{aligned}
$$

Используя условие эллиптичности операторов $L_{i}$, получаем из $(58)$

$$
\begin{gathered}
\int_{\Pi_{a, b}} \sum_{k=1}^{N} \sum_{i=1}^{n}\left|\frac{\partial}{\partial x_{i}}\left(u_{k}-\bar{u}_{k}\right)\right|^{2} d x \\
-\int_{x_{n}=b}\left[\sum_{k=1}^{N} u_{k 0}(\widehat{x})\left(u_{k}-\bar{u}_{k}\right) \frac{\partial}{\partial x_{n}}\left(u_{k}-\bar{u}_{k}\right)-\frac{1}{2} \sum_{k=1}^{N} a_{n}^{(k)} u_{k 0}(\widehat{x})\left(u_{k}-\bar{u}_{k}\right)^{2}\right] d \widehat{x} \\
+\int_{x_{n}=a} \sum_{k=1}^{N} u_{k 0}(\widehat{x})\left[\left(u_{k}-\bar{u}_{k}\right) \frac{\partial}{\partial x_{n}}\left(u_{k}-\bar{u}_{k}\right)\right] d x \\
\quad+\sum_{k=1}^{N} \int_{\Pi_{a, b}} u_{k 0}(\widehat{x})\left(u_{k}-\bar{u}_{k}\right)\left(\bar{f}_{k}-f_{k}\right) d x \leqslant 0
\end{gathered}
$$

Оценим последнее слагаемое в левой части неравенства (59). Для этого заметим, что из неравенства Пуанкаре следует

$$
\begin{aligned}
\int_{\Omega}\left|\bar{f}_{k}-f_{k}\right|^{2} d \widehat{x} & \leqslant c \int_{\Omega} \sum_{i=1}^{n-1}\left|\frac{\partial}{\partial x_{i}}\left(f_{k}\left(u, x_{n}\right)-\overline{f_{k}\left(u, x_{n}\right.}\right)\right|^{2} d \widehat{x} \\
& \leqslant c_{1} \int_{\Omega}\left|\nabla f_{k}\right|^{2}|\nabla u|^{2} \leqslant \varepsilon \int_{\Omega}|\nabla u|^{2} d x
\end{aligned}
$$


если $\rho$ в условии теоремы 4 достаточно мало. Из (60), (59) следует:

$$
\begin{aligned}
& \frac{1}{2} \int_{\Pi_{a, b}} \sum_{k=1}^{N} \sum_{i=1}^{n}\left|\frac{\partial}{\partial x_{i}}\left(u_{k}-\bar{u}_{k}\right)\right|^{2} d x \\
& -\int_{x_{n}=b}\left[\sum_{k=1}^{N} u_{k 0}(\widehat{x})\left(u_{k}-\bar{u}_{k}\right) \frac{\partial}{\partial x_{n}}\left(u_{k}-\bar{u}_{k}\right)-\frac{1}{2} \sum_{k=1}^{N} a_{n}^{(k)} u_{k 0}(\widehat{x})\left(u_{k}-\bar{u}_{k}\right)^{2}\right] d \widehat{x} \\
& \quad+\int_{x_{n}=a} \sum_{k=1}^{N} u_{k 0}(\widehat{x})\left[\left(u_{k}-\bar{u}_{k}\right) \frac{\partial}{\partial x_{n}}\left(u_{k}-\bar{u}_{k}\right)\right] d x \leqslant 0
\end{aligned}
$$

Из ограниченности $u_{k}(x)$ и из свойств решений линейных эллиптических уравнений следует, что

$$
\int_{\Pi_{t, t+1}}\left|\nabla u_{k}\right|^{2} d x \leqslant c
$$

где $c$ от $t$ не зависит.

Следовательно,

$$
\int_{\Pi_{t, t+1}}\left|\nabla \bar{u}_{k}\right|^{2} d x \leqslant c_{1}
$$

равномерно по $t$.

Отсюда следует, что если $b \rightarrow \infty$ по некоторой последовательности, то

$$
\frac{1}{2} \int_{\Pi_{a, b}} \sum_{k=1}^{N} \sum_{i=1}^{n}\left|\frac{\partial}{\partial x_{i}}\left(u_{k}-\bar{u}_{k}\right)\right|^{2} d x \leqslant c_{2} .
$$

Таким образом,

$$
\int_{\Pi_{a, \infty}} \sum_{k=1}^{N} \sum_{i=1}^{n}\left|\frac{\partial}{\partial x_{i}}\left(u_{k}-\bar{u}_{k}\right)\right|^{2} d x \leqslant c_{2} .
$$

Следовательно,

$$
\begin{aligned}
\lim _{m \rightarrow \infty} \int_{x_{n}=b_{m}}\left[\sum_{k=1}^{N} u_{k 0}(\widehat{x})\left(u_{k}-\bar{u}_{k}\right)\right. & \frac{\partial}{\partial x_{n}}\left(u_{k}-\bar{u}_{k}\right) \\
& \left.-\frac{1}{2} \sum_{k=1}^{N} a_{n}^{(k)} u_{k 0}(\widehat{x})\left(u_{k}-\bar{u}_{k}\right)^{2}\right] d x=0
\end{aligned}
$$

где $b_{m}$ некоторая последовательность, стремящаяся к $+\infty$.

Устремив в (61) $b$ к $\infty$ по последовательности $b_{m}$, получим в силу $(62)$

$$
\begin{aligned}
& \frac{1}{2} \int_{\Pi_{a, \infty}} \sum_{k=1}^{N} \sum_{i=1}^{n}\left|\frac{\partial}{\partial x_{i}}\left(u_{k}-\bar{u}_{k}\right)\right|^{2} d x \\
& \quad \leqslant-\int_{x_{n}=a} \sum_{k=1}^{N} u_{k 0}(\widehat{x})\left[\left(u_{k}-\bar{u}_{k}\right)-\frac{1}{2} \sum_{k=1}^{N} a_{n}^{(k)} u_{k 0}(\widehat{x})\left(u_{k}-\bar{u}_{k}\right)^{2}\right] d \widehat{x} .
\end{aligned}
$$


Отсюда и из неравенства Пуанкаре получаем

$$
J(a)=\int_{\Pi_{a, \infty}} \sum_{k=1}^{N} \sum_{i=1}^{n}\left|\frac{\partial}{\partial x_{i}}\left(u_{k}-\bar{u}_{k}\right)\right|^{2} d x \leqslant C \int_{x_{n}=a} \sum_{k=1}^{N} \sum_{i=1}^{n}\left|\frac{\partial}{\partial x_{i}}\left(u_{k}-\bar{u}_{k}\right)\right|^{2} d x
$$

т.е.

$$
J(t) \leqslant-\beta J^{\prime}(t)
$$

Следовательно, $J(t) \leqslant C e^{-\beta t}$. Используя еще раз неравенство Пуанкаре, получаeм:

$$
\int_{\Pi_{a}} \sum_{k=1}^{N} \sum_{i=1}^{n}\left|\frac{\partial}{\partial x_{i}}\left(u_{k}-\bar{u}_{k}\right)\right|^{2} d x+\int_{\Pi_{a}} \sum_{k=1}^{N}\left|u_{k}-\bar{u}_{k}\right|^{2} d x \leqslant c_{1} e^{-2 \beta a} .
$$

Перепишем систему уравнений (54) в виде:

$$
\frac{d}{d x_{n}^{2}} \bar{u}_{k}+a_{n}^{(k)} \frac{d}{d x_{n}} \bar{u}_{k}=f_{k}\left(x_{n} \bar{u}\right)-g_{k}
$$

где $g_{k}\left(x_{n}\right)=f_{k}\left(x_{n} \bar{u}\right)-\overline{f_{k}\left(x_{n} u\right)}, k=1, \ldots, N$. Легко видеть, что

$$
g_{k}\left(x_{n}\right)=f_{k}\left(x_{n} \bar{u}\right)-\overline{f_{k}\left(x_{n} u\right)}+\overline{f_{k}\left(x_{n} \bar{u}\right)}-\overline{f_{k}\left(x_{n} \bar{u}\right)}=\overline{f_{k}\left(x_{n} \bar{u}\right)-f_{k}\left(x_{n} u\right)} .
$$

Так как

$$
\left|f_{k}\left(x_{n} u\right)-f_{k}\left(x_{n} \bar{u}\right)\right| \leqslant\left\|\nabla_{u} f_{k}\right\||u-\bar{u}|
$$

и $u(x)$ - ограниченная функция, то

$$
\left|g_{s}\right| \leqslant c_{1} \int_{\Omega}|u-\bar{u}| d \widehat{x}
$$

и

$$
\left|g_{s}\right|^{2} \leqslant c_{2} \int_{\Omega}|u-\bar{u}|^{2} d \widehat{x}
$$

Из (63) следует, что

$$
\int_{x_{n}}^{\infty}\left|g_{s}\right|^{2} d t \leqslant c_{3} e^{-2 \beta x_{n}} .
$$

К системе (64) можно применить теорему 1 , из которой следует, что

$$
\bar{u}=X\left(x_{n}\right)+v_{1}\left(x_{n}\right)
$$

где $\left\|v_{1}\right\|=O\left(e^{-\alpha x_{n}}\right)$.

Отсюда и из (63) получаем утверждение теоремы 5.

Отметим, что в случае одного уравнения утверждение теоремы 5 оказывается более сильньм. Именно, в этом случае имеет место поточечная оценка:

$$
\|v\| \leqslant e^{-\alpha x_{n}} .
$$

Это доказывается так же, как теорема 3 для параболического уравнения с использованием оценок Де Джорджи-Мозера [4]-[6].

Неравенство (65) верно и при произвольном $N$, если потребовать некоторой гладкости коэффициентов $a_{i j}^{(k)}$. Достаточно, чтобы они удовлетворяли условию Гёльдера, а граница $\partial \Omega$ области $\Omega$ была бы ляпуновской поверхностью. В этом случае вместо оценок типа Де Джорджи-Мозера используются оценки Морри [7].

Приведем несколько примеров, где используется теорема 5. 
1. Рассмотрим уравнение

$$
\sum_{i, j=1}^{n} \frac{\partial}{\partial x_{i}} a_{i j}(\widehat{x}) \frac{\partial u}{\partial x_{j}}+\sum_{i=1}^{n-1} a_{i}(\widehat{x}) \frac{\partial u}{\partial x_{i}}+k \frac{\partial u}{\partial x_{n}}-a_{0}|u|^{\sigma-1} u=0
$$

где $\sigma>1, a_{0}=$ const $>0, a_{i j}, a_{i}$ удовлетворяют условиям (48), ограничены, измеримы в липшицевой ограниченной области $\Omega$.

Рассмотрим решение уравнения (66) с краевым условием (49) при $N=1$. Известно [8], что все такие решения имеют нулевой предел при $x_{n} \rightarrow+\infty$. Из теоремы 4 следует, что при $k=0$

$$
u=c_{\sigma}\left(x_{n}+T\right)^{\frac{2}{1-\sigma}}+O\left(e^{-\alpha x_{n}}\right),
$$

где $\alpha>0$ - постоянная, $T$ - постоянная, которая зависит от решения. Постоянная $c_{\sigma}$ или равна нулю, или $\left[\frac{2(1+\sigma)}{(1-\sigma)^{2}} a_{0}\right]^{\frac{1}{\sigma-1}}=\left|c_{\sigma}\right|$, причем $c_{\sigma}=0$ тогда и только тогда, когда решение меняет знак в каждой области $\Pi_{a}, a>0$.

Если $k<0$, то из теоремы 4 следует:

$$
u=\chi\left(x_{n}+T\right)+O\left(e^{-\alpha x_{n}}\right),
$$

где $\chi$-некоторое решение уравнения

$$
\ddot{\chi}+k \dot{\chi}-a|\chi|^{\sigma-1} \chi=0,
$$

ограниченное при $x_{n} \rightarrow+\infty$. Легко видеть, что значениями в нуле всякое ограниченное на $+\infty$ решение уравнения (68) определяется однозначно, и оно стремится к нулю при $x_{n} \rightarrow+\infty$. Если $\chi(0)=0$, то $\chi \equiv 0$. В формуле (67) $\chi \equiv 0$ в том и только том случае, когда $u(x)$ меняет знак в каждой области $\Pi_{a}, a>0$. Заметим, что если $\chi(0)>0$, то

$$
\chi=C\left(x_{n}+T\right)^{\frac{1}{1-\sigma}}+C_{1}\left(x_{n}+T\right)^{\frac{\sigma}{1-\sigma}} \ln \left(x_{n}+T\right)(1+o(1)),
$$

где $C=\left(\frac{k \lambda}{a_{0}}\right)^{\frac{1}{1-\sigma}}, \lambda=\frac{1}{1-\sigma}, C_{1}=\frac{C \lambda(1-\lambda)}{k}, T=$ const, которая зависит от $\chi$.

Наконец, если $k>0$, то $u(x)=O\left(e^{-\alpha x_{n}}\right)$, где $u$-любое решение задачи (66), (49) в $\Pi_{0}$.

2. Рассмотрим в $\Pi_{0}$ уравнение

$$
\Delta u+|u|^{\sigma-1} u=0, \quad \sigma>1,
$$

и его решение, которое удовлетворяет условиям (49). Докажем, что существует $h=$ const $>0$ такая, что если $|u(x)| \leqslant h$ при $x_{n}$ достаточно больших, то либо

$$
u=O\left(e^{-\alpha x_{n}}\right), \quad \alpha=\text { const }>0,
$$


либо

$$
u=\chi\left(x_{n}\right)+O\left(e^{-\alpha x_{n}}\right),
$$

где $\chi\left(x_{n}\right)$ - периодическая функция.

Действительно, из теоремы 4 следует, что

$$
u=\chi\left(x_{n}\right)+O\left(e^{-\alpha x_{n}}\right),
$$

где

$$
\ddot{\chi}+|\chi|^{\gamma-1} \chi=0 .
$$

Легко видеть, что все нетривиальные решения этого обыкновенного дифференциального уравнения периодичны.

Заметим, что уравнение (69) не имеет знакопостоянных решений в $\Pi_{0}$, удовлетворяющих условиям (49). Действительно, пусть $u(x)>0$ в $\Pi_{a}$. Тогда в силу принципа максимума $u(x) \geqslant \min _{x_{n}=a} u(x)=\gamma>0$. Из (69) следует:

$$
\frac{d^{2}}{d x_{n}^{2}} \bar{u} \leqslant-\gamma^{\sigma}
$$

откуда $\bar{u} \rightarrow-\infty$, когда $x_{n} \rightarrow+\infty$. Это является противоречием.

3. Рассмотрим в $\Pi_{0}$ уравнение:

$$
\Delta u+u-k|u|^{\sigma-1} u=0
$$

$\sigma=$ const $>1, k=$ const.

Допустим, что $u(x)$ - его решение, удовлетворяет условиям (49) и $\lim _{x_{n} \rightarrow \infty} u(x)=0$. Если $\lambda_{2}(\Omega)$ - наименьшее по модулю ненулевое собственное значение задачи Неймана для оператора Лапласа в $\Omega$ - больше 1 , то

$$
u(x)=O\left(e^{-\alpha x_{n}}\right) .
$$

Доказательство этого утверждения аналогично доказательству теоремы 3 и мы его приводить не будем.

4. Рассмотрим уравнение

$$
\Delta u-u_{+}^{\sigma}=0, \quad \sigma>1,
$$

где $u_{+}-$положительная часть функции $u$ в $\Pi_{0}$ и его решение, удовлетворяюшее однородному краевому условию Неймана на $\Gamma_{a}$. Пусть $\lim _{x_{n} \rightarrow \infty} u(x)=0$. Тогда

$$
u=c_{\sigma}\left(x_{n}+T\right)^{\frac{2}{1-\sigma}}+O\left(e^{-\alpha x_{n}}\right),
$$

где или $c_{\sigma}=\left[\frac{2(1+\sigma)}{(1-\sigma)^{2}}\right]^{\frac{1}{1-\sigma}}$, или $c_{\sigma}=0$.

Случай $c_{\sigma}=0$ возможен тогда и только тогда, когда $u(x)$ меняет знак в каждой области $\Pi_{a}, a>0$. Заметим (это следует из теории гармонических функций), что кроме решений, стремящихся к нулю на бесконечности, рассматриваемая задача может иметь еще только решения вида:

$$
u=C_{1} x_{n}+C_{2}+O\left(e^{-\alpha x_{n}}\right),
$$

где $C_{1}=$ const $<0$, постоянные $C_{1}, C_{2}$ зависят от $u$. 
5. Рассмотрим уравнение

$$
\Delta u+u_{+}^{\sigma}=0, \quad \sigma>1, \quad x \in \Pi_{0},
$$

и пусть $u(x)$ удовлетворяет однородному условию Неймана на $\Gamma_{0}$ и имеет предел нуль на бесконечности. Тогда

$$
u(x)=O\left(e^{-\alpha x_{n}}\right), \quad x_{n} \rightarrow+\infty .
$$

6. Рассмотрим уравнение

$$
\Delta u+i|u|^{\sigma-1} u=0, \quad \sigma>1,
$$

и его решение в $\Pi_{0}$, удовлетворяющее однородному краевому условию Неймана на $\Gamma_{0}$. Теорема 1 применима, конечно, и для комплекснозначных функций. Это сразу следует в результате разделения вешественной и мнимой частей. Используя ее, можно сделать утверждение, что если $\lim _{x_{n} \rightarrow+\infty} u=0$, то

$$
u=O\left(e^{-\alpha x_{n}}\right) .
$$

Приведем теперь примеры применения теоремы 1 для уравнений высокого порядка.

7. Рассмотрим уравнение:

$$
\frac{\partial u}{\partial t}=-\frac{\partial^{4} u}{\partial x^{4}}+a|u|^{\sigma-1} u+b|u|^{\tau-1} u
$$

где $\tau>\sigma>1$. Пусть $u(x, t)$ - решение этого уравнения, периодическое по $x$. Если $\lim _{t \rightarrow+\infty} u=0$, то

$$
u(t, x)=X(t)+O\left(e^{-\alpha t}\right), \quad \alpha>0,
$$

где $X(t)$ - решение уравнения

$$
\dot{X}(t)=\alpha X(t)^{\sigma}+b X(t)^{\tau} .
$$

Заметим, что если $b=0, a<0$, то всякое решение стремится к 0 при $t \rightarrow \infty$. Если $\alpha>0$, то только тривиальное решение имеет нулевой предел при $t \rightarrow \infty$.

8. Рассмотрим уравнение

$$
\Delta \Delta u=a|u|^{\sigma-1} u, \quad \sigma>1,
$$

и его решение $u(x)=u\left(x^{\prime}, x_{n}\right)$, периодическое по $x^{\prime}$.

Если $\lim _{x_{n} \rightarrow \infty} u(x)=0$, то

$$
u(x)=X\left(x_{n}\right)+O\left(e^{-\alpha x_{n}}\right), \quad \alpha>0,
$$

где $X\left(x_{n}\right)$ - решение уравнения

$$
X^{(4)}\left(x_{n}\right)=a|X|^{\sigma} X .
$$

Если $\alpha<0$, то все решения последнего уравнения колеблюшиеся (имеют неограниченную последовательность нулей при $\left.x_{n} \rightarrow \infty\right)$ и $\left|X\left(x_{n}\right)\right| \leqslant c x_{n}^{4 /(1-\sigma)}$. Кроме того

$$
\varlimsup_{x_{n} \rightarrow+\infty} x_{n}^{\frac{4}{\sigma-1}} X\left(x_{n}\right)>0 .
$$

Если $\alpha>0$, то $X\left(x_{n}\right) \sim c_{\sigma} x_{n}^{\lambda}$, где

$$
c_{\sigma}=\left[\frac{\lambda(\lambda-1)(\lambda-2)(\lambda-3)}{a}\right]^{1 /(\sigma-1)}, \quad \lambda=\frac{4}{1-\sigma} .
$$


9. Пусть $u(x)$ решение уравнения:

$$
\frac{\partial u}{\partial t}=-\frac{\partial^{4} u}{\partial x^{4}}+\frac{\partial^{2} u}{\partial x^{2}}+a|u|^{\sigma-1} u+b|u|^{\tau-1} u, \quad t>0, \quad 0<x<1,
$$

где $\sigma>1, \tau>1$ и его решение в $\Pi_{0}$, удовлетворяюшее краевым условиям:

$$
u^{\prime \prime}(0)=u^{\prime \prime}(1)=0, \quad \alpha^{2} u^{\prime \prime \prime}(0)-u^{\prime}(0)=\alpha^{2} u^{\prime \prime \prime}(1)-u^{\prime}(1)=0 .
$$

Такие условия можно считать аналогом краевых условий Неймана, что следует из вариационного принципа для стационарной задачи. Если $u$ имеет нулевой предел при $t \rightarrow+\infty$, то верна формула (71), где $X(t)$ решение уравнения (72). Отметим еще только, что в случае $a<0$ и $X(t) \rightarrow 0$ при $t \rightarrow \infty$ или

$$
X(t)= \pm\left[\frac{1}{(1-\sigma) a}\right]^{\frac{1}{1-\sigma}} t^{\frac{1}{1-\sigma}}(1+o(1)),
$$

или $X(t)=O\left(e^{-\alpha t}\right)$, где $\alpha>0$.

\section{Список литературы}

1. Kondratiev V.A., Oleinik O.A. Some results for nonlinear elliptic equations in cylindrical domains // Oper. Theory Adv. Appl. 1992. V. 57. P. 185-195.

2. Кондратьев B. А., Ландис E. M. О качественных свойствах решений одного нелинейного уравнения второго порядка // Матем. сб. 1988. Т. 138. №3. С. 346-359.

3. Ладыженская О.А., Уральцева Н. Н., Солонников В. А. Линейные и квазилинейные уравнения параболического типа. М.: Наука, 1967.

4. De Giorgi E. Sulla differenziabilita e l'analiticita delle estremali degli integrali multipli regolari. // Mem. Accad. Sci. Torino (3). P. I., III. 1957. P. 25-43.

5. Moser J. A new proof of de Giorgi theorem concerning the regularity problem for elliptic differential equations // Comm. Pure Appl. Math. 1960. V. 13. №3. P. 457-468.

6. Ладъљенскал О. А., Уральцева Н. Н. Линейные и квазилинейные уравнения эллиптического типа. М.: Наука, 1964.

7. Morrey G. B. Multiple Integrals in the Calculus of Variations. Berlin: Springer-Verlag, 1966.

8. Kondratiev $V$., Oleinik $O$. On asimptotic behaviour of solutions of some nonlinear elliptic equations in unbounded domains // Partial differential equations and related subjects. Proceedings of the Conference dedicated to Louis Nirenberg. Longman, 1992. P. 163-195.

Московский государственньй

Поступила в редакцию

университет им. М.В. Ломоносова

16.06.1997 